\title{
Decreased Functional Caspase-3 Expression in Umbilical Cord Blood Neutrophils Is Linked to Delayed Apoptosis
}

\author{
DEFANG LUO, KENNETH O. SCHOWENGERDT, JR, JOSEPH J. STEGNER, \\ W. STRATFORD MAY, JR, AND JOYCE M. KOENIG \\ Department of Pediatrics [D.L., K.O.S., J.J.S., J.M.K], Department of Medicine [W.S.M.], Shands Cancer \\ Center [W.S.M.], University of Florida College of Medicine, Gainesville, Florida, U.S.A.
}

\begin{abstract}
ABST
Resolution of inflammatory processes depends on the efficient
removal of aging neutrophils by the reticuloendothelial system.
Neutrophil apoptosis is key to this process, and its impairment
may contribute to the pathogenesis of chronic inflammation. We
recently discovered that Fas-mediated apoptosis in umbilical
cord blood neutrophils was significantly delayed as compared
with those of adults. Because execution of apoptosis relies on
caspases, we used reverse transcription PCR, immunoblots, and
enzymatic assays to study the integrity of several members of
those proteases known to mediate Fas-induced apoptosis in
neutrophils. Our results indicate that diminished expression of
caspase-3 mRNA and the precursor form of the protein, as well
\end{abstract}
Neutrophil apoptosis contributes to the homeostasis of the immune system through its role in the resolution of inflammation (1). During this process, tissue macrophages scavenge aging neutrophils while preserving the integrity of surrounding tissue and minimizing local injury (2). Anti-inflammatory cytokines, such as IL-10, promote the resolution of inflammation partly through their induction of neutrophil apoptosis (3). In contrast, pro-inflammatory cytokines, including granulocyte colony-stimulating factor, can delay the onset of neutrophil apoptosis (4). Although this action might benefit the host during active infection by prolonging the survival of bactericidal neutrophils, the persistence of neutrophils after delayed apoptosis in the absence of an infectious focus may also lead to subsequent localized injury (5). Such a scenario has been thought to precede a variety of inflammatory disorders, including rheumatoid arthritis (6) and the acute respiratory distress syndrome in adults (7). Similarly, a diminished capacity to

Received May 7, 2002; accepted November 15, 2002.

Correspondence: Joyce M. Koenig, M.D., Department of Pediatrics, University of Florida College of Medicine, 1600 Archer Rd., RG 130, Gainesville, FL 32610-0296, U.S.A.; e-mail: koenijm@peds.ufl.edu

Supported, in part, by grants from the National Institutes of Health (HD01062, HD41559, and Clinical Research Center Grant RR00082) (J.M.K.), and a grant from the Howard Hughes Medical Institute Research Resources Program (J.M.K.).

DOI: 10.1203/01.PDR.0000059747.52100.2E as a lower functional enzymatic activity of caspase-3, correlates with delayed apoptosis in umbilical cord blood neutrophils. Our data suggest that functional expression of caspase-3 in neutrophils may be regulated during ontogeny. (Pediatr Res 53: 859864,2003 )
Ab, antibody
Abbreviations
AFC, 7-amino-4-trifluoremethyl coumarin
FasL, Fas ligand
pNA, $p$-nitroanilide
RT, reverse transcription

resolve inflammation could potentially contribute to the pathogenesis of bronchopulmonary dysplasia, a chronic inflammation of the lungs frequently observed in premature infants.

The apoptotic pathway involving interactions between the surface antigen, Fas/CD95, and its ligand, FasL, seems to play a significant role in neutrophil apoptosis $(8,9)$. Neutrophils have a higher expression of Fas than do other leukocytes (9), and may contribute to their own apoptosis in an autocrine manner through their release of FasL. Interaction of Fas with FasL or with agonistic anti-Fas antibody activates the FADD (Fas-associated death domain protein)/MORT1 complex (10), which results in proteolytic cleavage of the inactive procaspase- 8 protein into the active enzyme. This activation initiates a hierarchy of cleavage reactions of downstream caspases, including caspase-3 (11). Caspases mediate their apoptotic effects by activating specific substrates, including those involved in regulating the cell cycle and in signaling mechanisms (12). The evolutionary importance of caspases in the regulation of apoptosis is emphasized by the functional homology between ced-3, the "death" gene that controls apoptosis during embryonic development of Caenorhabiditis elegans, and members of the IL- $1 \beta$ converting enzyme-like family of caspases (13). Recent evidence has shown the pivotal involvement of caspase- 8 and caspase- 3 
in mediating early apoptotic events after activation of Fas $(11,14)$.

We have previously observed that, compared with neutrophils of adults, cord blood neutrophils have a delayed progression of apoptotic events mediated by the Fas/FasL pathway as well as spontaneous apoptosis (15). The resistance of cord blood neutrophils to Fas-mediated apoptosis did not seem to be the result of differential protein expression of Fas antigen or of Fas, indicating either that initial Fas activation itself, or that apoptotic events downstream of this activation, differed between cord blood and adult neutrophils (15). In addition, our observation that cycloheximide, a protein synthesis inhibitor, induced less apoptosis in cord blood neutrophils than in those of adults suggested the preexistence of anti-apoptotic proteins, the diminished expression of proteins that promote apoptosis in cord blood neutrophils, or both (15). To begin to delineate the mechanisms underlying these observations, we designed studies to test the hypothesis that delayed apoptosis in cord blood neutrophils is related to a lower expression of apoptotic proteins with actions that target events downstream of initial Fas activation. In this article, we report that umbilical cord blood neutrophils have lower expression of a cysteine protease central to Fas-mediated apoptosis, caspase-3, than do those of adults, and that diminished caspase- 3 function is linked to delayed apoptosis in those neutrophils.

\section{METHODS}

Neutrophil isolation and culture. Paired samples obtained from the umbilical veins of term placentas after uncomplicated cesarean delivery or from the peripheral venous blood of healthy adult volunteers were processed immediately after collection. Samples were obtained with informed consent in accordance with the guidelines of the Institutional Review Board for Human Studies at the University of Florida.

Dextran-sedimented neutrophils were subjected to density centrifugation followed by hypotonic lysis of contaminating erythrocytes (16). The purity of neutrophil suspensions was $>95 \%$ (morphologic evaluation); viability was $>95 \%$ (trypan blue exclusion). Isolated neutrophils $\left(2 \times 10^{6} / \mathrm{mL}\right)$ suspended in endotoxin-free RPMI 1640/5\% FCS (Invitrogen, Carlsbad, CA, U.S.A.) were cultured in polypropylene tubes at $37^{\circ} \mathrm{C}, 5 \%$ $\mathrm{CO}_{2}$ for up to $48 \mathrm{~h}$, and harvested for determination of the apoptotic fraction by terminal deoxynucleotidyl transferase (TdT)-mediated dUTP-biotin nick-end labeling (TUNEL) assay or for measurement of caspase activity. To evaluate the induction of Fas-mediated apoptosis, in some studies neutrophils were cultured in the presence of anti-human Fas IgM antibody (300 ng/mL; clone CH11, Upstate Biotechnology, Lake Placid, NY, U.S.A.) to induce apoptosis, as we have done previously (15).

RT-PCR analysis of caspase expression. Total RNA was extracted from lysates of freshly isolated cord blood or adult neutrophils $\left(10^{7}\right.$ cells) using a commercial kit (RNeasy Mini Kit, QIAGEN, Valencia, CA, U.S.A.), and reverse transcription was performed using oligo(dT) priming as has been described (17). Primer pairs were synthesized based on published sequences (caspase-1, caspase-3) (18); or were derived from an EMBL/GenBank sequence (caspase-8) (19) using computer analysis software (Primer Designer, Scientific \& Educational Software, Durham, NC, U.S.A.). Primer pairs for caspase-8 were as follows: 5' primer, CACGATGACTGCACAGTAG; 3' primer, CTTCAGTCAGGATGGTGAG. Each PCR reaction was subjected to 35 cycles of specific amplification conditions. The PCR cycling conditions for caspase-3, caspase- 8 , and glyceraldehyde-3-phosphate dehydrogenase (GAPDH) were: $1 \mathrm{~min}$ at $95^{\circ} \mathrm{C}$ for denaturing, $40 \mathrm{~s}$ at $61^{\circ} \mathrm{C}$ for annealing, and $45 \mathrm{~s}$ at $72^{\circ} \mathrm{C}$ for extension. For caspase-1, amplification conditions were as follows: $40 \mathrm{~s}$ at $94^{\circ} \mathrm{C}$ for denaturing, $35 \mathrm{~s}$ at $56^{\circ} \mathrm{C}$ for annealing, and $1 \mathrm{~min}$ at $72^{\circ} \mathrm{C}$ for extension. In addition, $2 \mathrm{~min}$ at $95^{\circ} \mathrm{C}$ for denaturing were added before the cycling period, and $5 \mathrm{~min}$ for extension were added at the end of the cycling period. Amplification reactions were carried out using a PTC-200 Peltier thermal cycler (MJ Research Co., Watertown, MA, U.S.A.), and the PCR products (10 $\mu \mathrm{L}$ per well) were run on a $2 \%$ agarose gel and visualized by UV light. Band density was determined using the Windowsbased program Scion Image (Scion Corp., Frederick, MD, U.S.A.) and a relative band density ratio of target gene to GAPDH was calculated.

Western blots. Cell lysates were prepared by resuspending pelleted neutrophils or positive control cells (THP.1) in lysis buffer $(50 \mathrm{mM}$ Tris-HCl, $\mathrm{pH} 7.4$, containing $1 \%$ Nonidet P-40, $0.25 \%$ sodium deoxycholate, and $150 \mathrm{mM} \mathrm{NaCl} ; 1 \mathrm{mM}$ each of EGTA, phenylmethylsulfonyl fluoride, $\mathrm{Na}_{3} \mathrm{VO}_{4}$, and $\mathrm{NaF}$; and $1 \mu \mathrm{g} / \mathrm{mL}$ each of aprotinin, leupeptin, and pepstatin), as described (20). Lysates were centrifuged at 14,000 rpm (16,000 $\times g$ ) for 15 min (Eppendorf Microcentrifuge, Brinkmann Instruments, Westbury, NY, U.S.A.) at $4^{\circ} \mathrm{C}$, and supernatant aliquots were stored at $-70^{\circ} \mathrm{C}$ until analysis. For each Western assay, lysate samples $(20-40 \mu \mathrm{g}$, measured by protein assay, Bio-Rad, Richmond, CA) were subjected to SDS-PAGE (10\% gel) under reducing conditions. The separated proteins were electrophoretically transferred to an Immobilon-P membrane (Millipore Corporation, Bedford, MA). The transfer membrane was blocked overnight in a $2 \%$ milk/TBS (TBS: $50 \mathrm{mM}$ Tris, $200 \mathrm{nM} \mathrm{NaCl}, \mathrm{pH} 7.5$ ) solution, then incubated in the presence of the following antibodies (1:1000 dilution): anti-caspase-3 (clone 19, Transduction Laboratories, Lexington, KY, U.S.A.); anti-caspase-1 (clone Ab-1, Calbiochem, San Diego, CA, U.S.A.) and anti-caspase-8 (clone 5F7, MBL Co., Nagoya, Japan). For protein detection, the washed membrane was incubated with a secondary $\mathrm{Ab}$, horseradish peroxidaseconjugated goat or rabbit anti-mouse $\mathrm{Ab}(1: 10,000)$ in blocking buffer ( $1 \mathrm{~h}, \mathrm{RT})$, followed by a 5 -min incubation with SuperSignal West Pico substrate (Pierce Chemical, Rockford, IL, U.S.A.). The treated membrane was exposed to autoradiographic film (CLXPosure Film, Pierce) for up to $5 \mathrm{~min}$. Band analysis by densitometry was performed for each sample (Scion), and the relative band density ratio of target protein to an internal standard (Jurkat or THP.1 cells) was calculated for each assay.

Measurement of relative caspase activity. A colorimetric assay was used (ApoAlert CPP32 Kit, Clontech, Palo Alto, CA, U.S.A.) to measure relative enzymatic activity of caspase- 3 in neutrophils cultured for $0-48 \mathrm{~h}$. This assay is based on spectrophotometric detection of the conjugated chro- 
mophore, pNA, after its cleavage from the labeled substrate specific for caspase-3, DEVD-pNA. Protease activity is determined in cell lysates by comparing the amount of absorbance from an apoptotic sample with an uninduced control, using a standard curve. Briefly, neutrophil aliquots $\left(2 \times 10^{6}\right)$ were lysed and the supernatants assayed using conjugated substrate (1 mM DEVD-pNA), or in its absence for control reactions, as per the manufacturer's instructions. Specificity of the reaction for caspase-3 was confirmed by adding the inhibitor, DEVDfmk, before the addition of the substrate. For spectrophotometric analysis, samples were transferred to 96-well plates (Nunc, Naperville, IL, U.S.A.), and the colorimetric reaction assayed at $405 \mathrm{~nm}$. Quantification of relative protease activity in OD units was determined from a calibration curve generated using standard concentrations of $\mathrm{pNA}$, the product resulting from cleavage of DEVD-pNA. This cleavage product is directly proportional to enzymatic activity of caspase-3.

For determination of relative caspase- 8 activity (ApoAlert caspase- 8 fluorometric assay kit, Clontech), a similar procedure was used with the following exceptions: 1) a specific caspase- 8 specific inhibitor (IETD-fmk) was used to establish specificity of the reaction for caspase- $8 ; 2$ ) caspase- 8 substrate, IETD, was conjugated to AFC; and 3 ) relative protease activity of cell lysates, measured by fluorescent emission of AFC, was determined by using a fluorometer with a 400-nm excitation filter and 505-nm emission filter. Quantification of relative protease activity in fluorescent units was determined from a calibration curve of AFC standards.

TUNEL assay. A commercial TUNEL assay (In-Situ Death Detection Kit, Roche Molecular Biochemicals, Indianapolis, IN, U.S.A.) was used to detect and quantitate apoptosis in neutrophil aliquots, as we have done previously (15). The mean channel fluorescence of labeled cells was determined by flow cytometric analysis of at least 10,000 events (FACScan; BD Biosciences, Franklin Lakes, NJ, U.S.A.).

Statistical analysis. Where appropriate, data are presented as the mean \pm SD. Results from paired experiments were evaluated for significance with the use of student's $t$ test or the one-way ANOVA; multiple pairwise comparisons were analyzed using the Student-Newman-Keuls test. A $p$ value $<0.05$ was considered to be significant.

\section{RESULTS}

Cord blood neutrophils have diminished expression of caspase-3 $\boldsymbol{m R N A}$. To determine whether delayed apoptosis in umbilical cord blood neutrophils might be the result of a lower expression of pro-apoptotic genes, we first studied these neutrophils for their expression of several caspases with key roles in Fas-mediated apoptosis. Shown in Figure 1 are gels representative

GAPDH

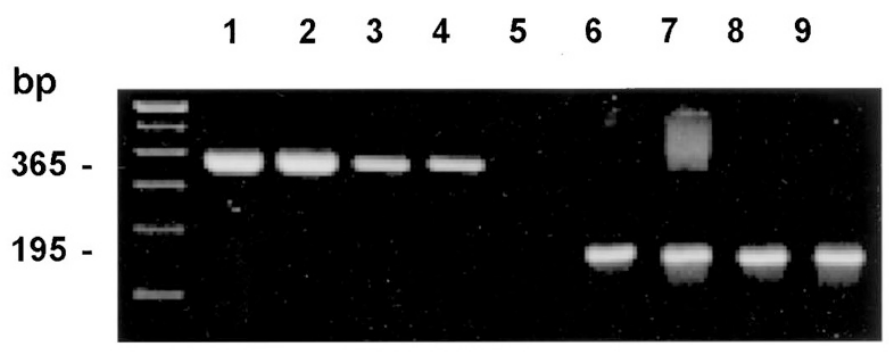

B

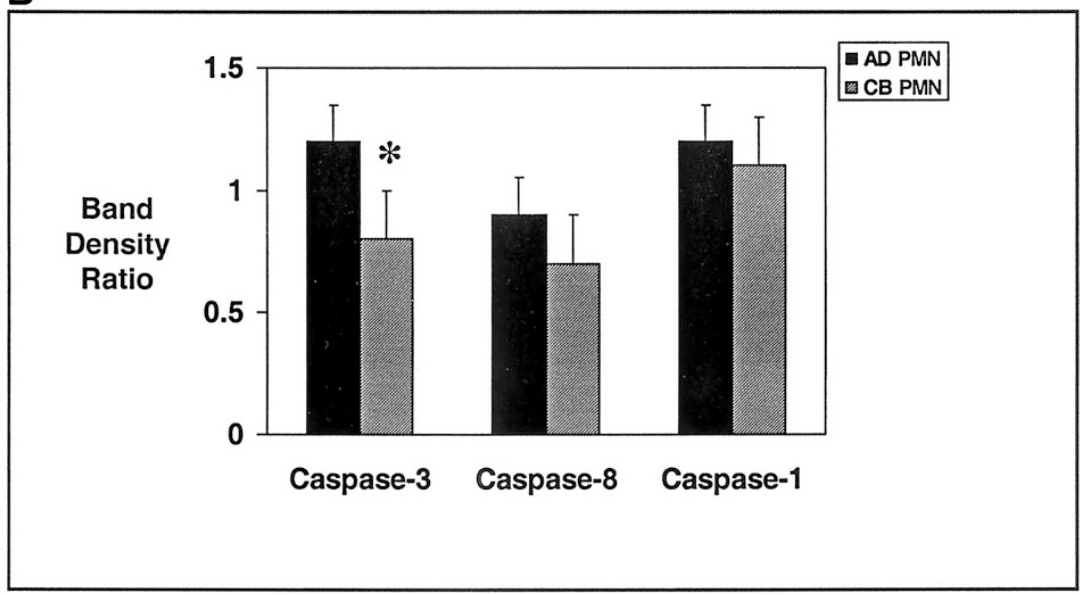

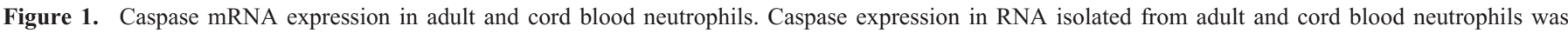

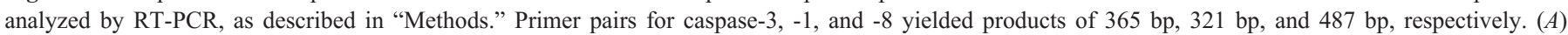

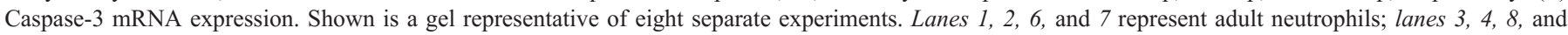

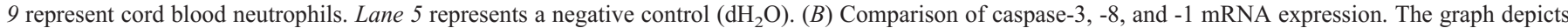

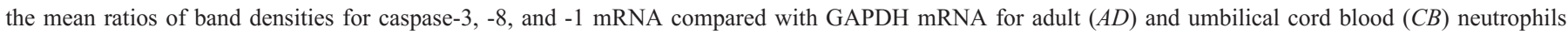
$(P M N)$. Error bars represent mean $\pm \mathrm{SD} .{ }^{*} p<0$. 01, AD vs CB PMN. 
of eight separate paired experiments. The caspase-3 (Fig. 1A) primer pair yielded a 365-bp product as previously reported (18). As shown in Figure $1 B$, band density ratios of mRNA for caspase-3 to GAPDH were lower in freshly isolated neutrophils from umbilical cord blood $(0.86 \pm 0.28)$ than in the peripheral blood of adults $(1.16 \pm 0.43, p<0.01)$. Although caspase- 8 expression tended to be lower in cord blood neutrophils $(0.70 \pm$ 0.22 versus adult neutrophils, $0.87 \pm 0.04$ ), this difference did not reach statistical significance. In contrast, band density ratios for caspase-1 (cord blood, $1.13 \pm 0.23$ versus adult, $1.10 \pm 0.23$; mean $\pm \mathrm{SD} ; p=\mathrm{NS}$ ) were similar between groups.

Cord blood neutrophils have less protein expression of procaspase-3. Shown in Figure 2 are Western blots representative of six separate assays. Densitometric band analysis showed a lower expression of the inactive $32 \mathrm{kD}$ precursor, procaspase-3, in freshly isolated cord blood neutrophil lysates $(0.39 \pm 0.21$ versus adults, $0.73 \pm 0.11 ; p<0.01)$, as well as in cord blood neutrophils cultured for $24 \mathrm{~h}(0.45 \pm 0.03$ versus adults, $0.58 \pm 0.08 ; p<0.05)$. In addition, expression of procaspase- 3 tended to be lower in adult neutrophils at $24 \mathrm{~h}$ compared with its expression at $0 \mathrm{~h}(p=0.06)$, consistent with the progressive loss of inactive caspase- 3 observed in aging neutrophils (21). In contrast, the amount of procaspase-3 protein in cord blood neutrophils at $0 \mathrm{~h}$ and $24 \mathrm{~h}$ did not differ ( $p$ $=$ NS). No differences were observed in the expression of procaspase-1 (cord blood, $0.46 \pm 0.09$ versus adult, $0.34 \pm$ $0.01 ; p=\mathrm{NS}$ ) and procaspase-8 (cord blood, $0.47 \pm 0.29$ versus adult, $0.38 \pm 0.24 ; p=\mathrm{NS}$ ), data not shown.

Cord blood neutrophils have less relative enzymatic activity of caspase-3 that correlates with a delayed progression of apoptosis. We next sought to determine whether a lower expression of caspase-3 correlated with diminished enzymatic activity. Neutrophils from umbilical cord blood and from adults were cultured for up to $48 \mathrm{~h}$ under varying conditions. In one set of experiments, neutrophils were cultured in media alone $(6 \mathrm{~h}, 24 \mathrm{~h}$, $48 \mathrm{~h})$ or in the presence of anti-Fas IgM (300 ng/mL) for $6 \mathrm{~h}$. As shown in Table 1, a greater fold increase in the amount of cleavage product (the chromophore, pNA), a relative measure of caspase-3 protease activity, was observed in adult neutrophils in

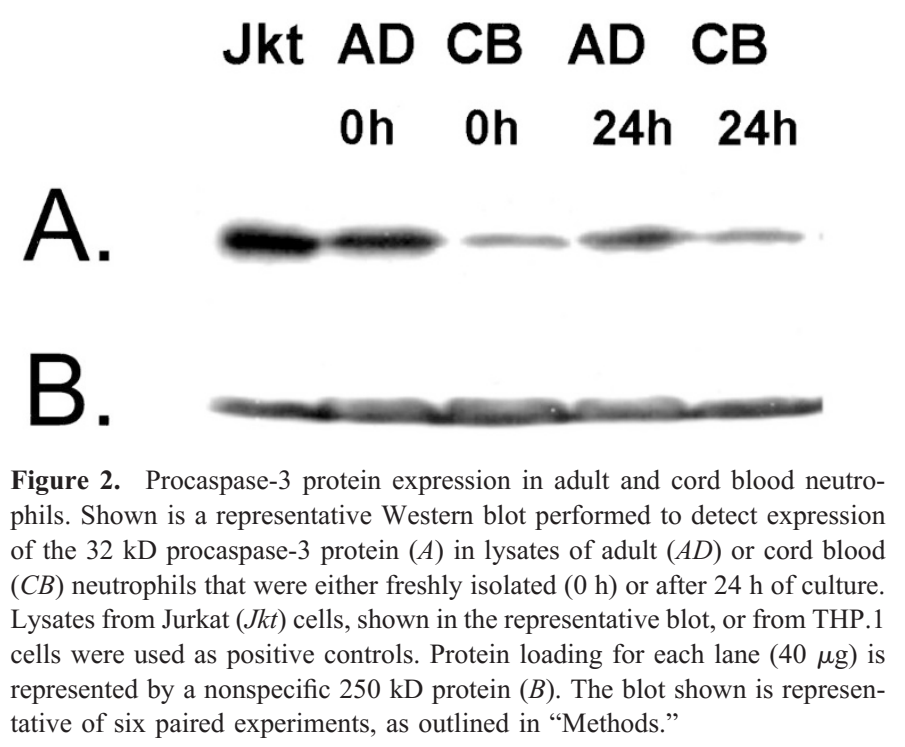

Table 1. Fold increase in relative caspase-3 activity in adult and cord blood neutrophils after Fas stimulation

\begin{tabular}{lllll}
\hline & \multicolumn{4}{c}{$6 \mathrm{~h}$} \\
& \multicolumn{1}{c}{$6 \mathrm{~h}$} & \multicolumn{1}{c}{$(+$ Fas $)$} & \multicolumn{1}{c}{$24 \mathrm{~h}$} & \multicolumn{1}{c}{$48 \mathrm{~h}$} \\
\hline Adult PMN & $1.2 \pm 0.1^{*}$ & $1.5 \pm 0.3^{*}$ & $2.5 \pm 0.3^{* *}$ & $0.8 \pm 0.4^{*}$ \\
Cord blood PMN & $1.0 \pm 0.1$ & $1.0 \pm 0.1$ & $1.6 \pm 0.2$ & $1.1 \pm 0.2$ \\
\hline
\end{tabular}

A colorimetric assay was utilized to measure the time-dependent increase in caspase-3 activity of cord blood and adult neutrophils cultured in the presence or absence of the apoptosis agonist, anti-Fas IgM Ab $(300 \mathrm{ng} / \mathrm{mL})$. Results for each time point are expressed as fold increase in protease activity over that measured at $0 \mathrm{~h}$. Data are representative of three separate paired experiments performed in duplicate. PMN, polymorphonuclear neutrophils. ${ }^{*} p<0.05$, ** $p<0.001$ vs cord blood neutrophils.

the presence of Fas agonist, or in its absence ( $24 \mathrm{~h}$ ) compared with cord blood neutrophils. In both groups, the increase in cleavage product over baseline values was greatest at $24 \mathrm{~h}$ of culture The amount of cleavage product decreased over the next $24 \mathrm{~h}$, and at $48 \mathrm{~h}$ was greater in cord blood neutrophils. In a second set of experiments that examined time-dependent spontaneous apoptosis, relative caspase-3 activity was greater for adult than cord blood neutrophils, peaking at $24 \mathrm{~h}$ of culture. At $48 \mathrm{~h}$, caspase activity was greater in cord blood neutrophils than in those of adults, but lower than at $24 \mathrm{~h}$ for both groups (Fig. 3).

In concurrent experiments, we used the TUNEL assay to correlate time-dependent relative caspase- 3 activity with the degree of spontaneous apoptosis in cultured neutrophils. We determined that cord blood and adult neutrophils cultured for up to $48 \mathrm{~h}$ showed differences in the progression of apoptosis (Fig. 4), similar to those we previously reported (15). The increasing proportion of apoptotic neutrophils in both groups paralleled the increases in relative caspase-3 activity at up to $24 \mathrm{~h}$ of culture (Fig. 3), and after $6 \mathrm{~h}$ of culture was greater for adult than for cord blood neutrophils. In contrast to caspase- 3 activity, the relative protease activity of caspase-8 (Fig. 5), another key component of the Fas/FasL pathway, was at least

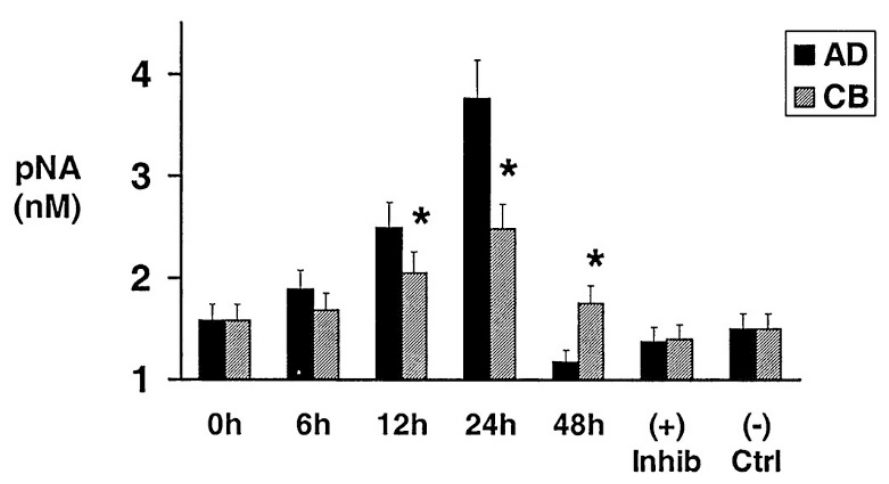

Figure 3. Enzymatic activity of caspase-3 in adult and cord blood neutrophils. Lysates were prepared from cultured aliquots of adult $(A D)$ and cord blood $(C B)$ neutrophils in four separate paired experiments performed in duplicate. Using a standard curve and a colorimetric assay, relative protease activity was determined by measuring the amount of pNA resulting from cleavage of the conjugated substrate (DEVD-pNA). The graph depicts mean values $( \pm \mathrm{SD})$ obtained from three separate paired experiments, with each timed sample assayed in duplicate. Also shown are enzymatic activity in neutrophils cultured for $24 \mathrm{~h}$ in the presence of the specific inhibitor, DEVD$\mathrm{CHO}[(+)$ Inhib] and activity in the absence of substrate [negative control, (-) Ctrl]. ${ }^{*} p<0.05$, adult $v s$ cord blood neutrophils. 


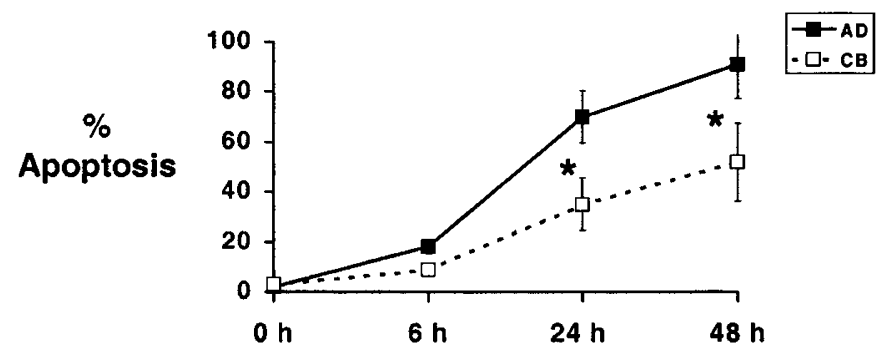

Figure 4. Spontaneous apoptosis in adult and cord blood neutrophils. Using the same samples as for the caspase-3 assay (Fig. 3), paired aliquots of adult $(A D)$ or cord blood $(C B)$ neutrophils, cultured for up to $48 \mathrm{~h}$, were stained for determination of apoptotic cells by the TUNEL assay. The graph depicts the mean proportion $( \pm \mathrm{SD})$ of apoptotic neutrophils in duplicate samples at specific time points, and is representative of three separate experiments. ${ }^{*} p<$ 0.02 , adult $v s$ cord blood neutrophils.

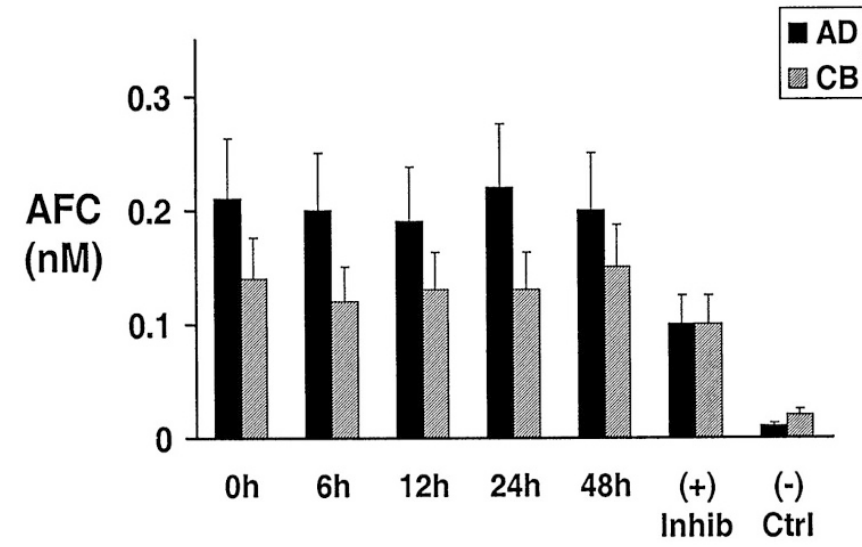

Figure 5. Enzymatic activity of caspase-8 in adult and cord blood neutrophils. Lysates were prepared from cultured aliquots of adult $(A D)$ and cord blood $(C B)$ neutrophils in three separate paired experiments performed in duplicate. Using a standard curve and a fluorometric assay, relative protease activity was determined by measuring the amount of AFC resulting from cleavage of the conjugated substrate (IETD-AFC). The graph depicts mean values $( \pm \mathrm{SD})$ obtained from three separate paired experiments, with each timed sample assayed in duplicate. Also shown are enzymatic activity in neutrophils cultured for $24 \mathrm{~h}$ in the presence of the specific inhibitor, IETD-fmk [(+) Inhib] and activity in the absence of substrate [negative control, $(-) \mathrm{Ctrl}$ ].

10-fold lower (0.2-0.4 $\mathrm{nM}$ of cleavage product) than that determined for caspase-3 at all time points measured, with no significant differences detected between cord blood and adult neutrophils at any one time point (Fig. 5). However, when values over time were compared between groups, cord blood neutrophils had less measurable caspase- 8 activity than did adult neutrophils during the same time period $(p<0.01)$.

\section{DISCUSSION}

In the present study, we determined that umbilical cord blood neutrophils from healthy term gestations have a lower functional expression of a critical Fas-associated cysteine protease, caspase-3, than do neutrophils from adult controls. Specifically, we observed that diminished expression of caspase-3 in cord blood neutrophils was linked to a lower enzymatic activity of caspase- 3 under conditions also associated with a delay in spontaneous apoptosis (15).

Our present findings are similar to those observed in studies comparing undifferentiated HL-60 cells with those induced toward granulocytic maturation (20). In those studies, differentiated HL-60 cells became susceptible to the induction of apoptosis by anti-Fas antibody, whereas the undifferentiated HL-60 cells were resistant, an observation reminiscent of our previous studies of apoptosis in cord blood and adult neutrophils (15). In addition, undifferentiated HL-60 cells were found to have less expression of caspase- 3 than their more mature counterparts, similar to our present findings in cord blood neutrophils. Furthermore, our observation that lower gene expression of caspase- 3 in cord blood neutrophils is associated with delayed enzymatic function parallels similar observations in HL-60 cells (20). However, only a relative comparison between terminally differentiated neutrophils and HL-60 cells is possible because, for example, neutrophils from adult subjects may lack several caspase substrates that are present in differentiated HL-60 cells, including the DNA repair enzymes, PARP and DNA-PK (21).

Alternative to the possibility that our observations in cord blood neutrophils reflect differences in their maturational stage, studies in other cell types suggest that ontogeny may also influence neutrophil apoptosis. For example, a recent study in rodents showed up-regulation of a novel suppressor of a $\mathrm{bcl}-$ 2-related gene during specific stages of lung development (22). Similarly, expression of Fas-associated genes appears to be agespecific in lymphocytes, and is linked with decreased apoptosis of cord blood T-cell subsets (23). In addition, the recently characterized caspase-14 is preferentially expressed in embryonic tissues, whereas it is absent from tissues of adults (24).

Caspases execute the apoptosis program in a number of cells, and evidence supports a major role for caspase-3 in mediating neutrophil apoptosis $(11,14,21)$. Pertinently, neutrophil apoptosis is delayed in caspase-3-deficient mice (25), although the exact mechanism underlying this observation has not been delineated. In addition, decreased caspase- 3 expression has been linked to delayed apoptosis of inflammatory lung neutrophils (26). The dependence of neutrophil apoptosis on caspase- 3 activity is underscored by the observation that neutrophils have minimal expression of caspase-7 (27), another key effector of Fas-mediated apoptosis (11). Thus, to undergo apoptosis, neutrophils may be unable to compensate for low caspase-3 activity by alternatively using caspase-7 (28). Because caspase- 3 is a pivotal component of the Fas/FasL pathway of apoptosis, these observations and our present data implicate the contribution of low caspase- 3 to the delayed apoptosis of cord blood neutrophils (15). This possibility is further supported by the observation that the onset of caspase- 3 enzymatic activity in aging neutrophils is coincident with protein and morphologic markers of apoptosis (21), as well as by our present observations showing that caspase-3 activity parallels the progression of apoptosis.

In the extrinsic pathway of apoptosis, the Fas/FasL pathway initiates an apoptotic cascade that involves activation of caspase- 8 followed by cleavage of procaspase-3 (11). Thus, one trivial explanation for the decreased caspase activity in cord blood neutrophils would include decreased Fas protein expression, although our previous studies showing similar Fas expression in adult and cord blood neutrophils suggest either that Fas activation itself or Fas-mediated events downstream of that activation are 
contributory (15). Alternatively, a deficiency of a major effector of apoptosis, such as caspase- 8 , might be expected to contribute to a delay in the progression of apoptosis triggered by death receptors. However, we did not detect appreciable differences in caspase-8 expression between cord blood and adult neutrophils in the present studies, although our studies suggested that enzymatic activity was lower in cord blood neutrophils. Caspase- 3 can also be activated independently of caspase- 8 by substrates that include PARP and DNA-PK, although their importance in mature neutrophils is unclear (21). In addition, recent studies have shown that apoptosis mediated by the mitochondrial pathway is associated with cytochrome $c$-induced activation of caspase-9, followed by processing of caspase-3 (29). Neutrophil apoptosis is associated with a decline in cytoskeletal functions (30), and thus might be regulated by cytoskeletal factors that also function as caspase substrates, such as focal adhesion kinase (31) and D4-GDPdissociation inhibitor (32). Gelsolin, another major cytoskeletal component, is a specific substrate for caspase-3 $(27,33)$, as well as a key intermediate in actin polymerization (34). Pertinently, actin polymerization activity is diminished in cord blood neutrophils (35), although whether gelsolin expression is also low in these neutrophils remains to be determined. Cleavage of the cytoskeletal element, fodrin (nonerythroid spectrin), a process linked to the early apoptotic externalization of phosphatidylserine on the outer plasma membrane (36), also seems to be mediated by caspase-3 (37). Thus, altered expression of these or other substrates, in addition to low protein expression of caspase-3, could potentially influence caspase- 3 activity in cord blood neutrophils.

In summary, we have determined that caspase-3, a key effector of the Fas/FasL pathway, has low functional expression in cord blood neutrophils. The findings outlined in the present article begin to delineate the mechanisms that underlie our previous observations of a delayed progression of apoptosis in cord blood neutrophils, and suggest that a deficiency of caspase- 3 is contributory. We speculate that a delay of neutrophil apoptosis in neonates could be involved in their impaired capacity to resolve infectious and inflammatory processes.

Acknowledgments. The authors thank the Clinical Research Center nurses, Pam Connolly, RN, and Ann Cothran, RN, and the obstetrical staff at Shands Hospital at the University of Florida for their help in acquiring cord blood samples. We also thank Sean Kuntz for his helpful technical support.

\section{REFERENCES}

1. Ellis RE, Yuan J, Horvitz HR 1991 Mechanisms and functions of cell death. Annu Rev Cell Biol 7:663-698

2. Haslett C, Savill JS, Whyte MK, Stern M, Dransfield I, Meagher LC 1994 Granulocyte apoptosis and the control of inflammation. Philos Trans R Soc Lond B Biol Sci 345:327-333

3. Cox G 1996 IL-10 enhances resolution of pulmonary inflammation in vivo by promoting apoptosis of neutrophils. Am J Physiol 271:L566-L571

4. Leavey PJ, Sellins KS, Thurman G, Elzi D, Hiester A, Silliman CC, Zerbe G, Cohen JJ, Ambruso DR 1998 In vivo treatment with granulocyte colony-stimulating factor results in divergent effects on neutrophil functions measured in vitro. Blood 92:4366-4374

5. Kasahara Y, Iwai K, Yachie A, Ohta K, Konno A, Seki H, Miyawaki T, Taniguchi N 1997 Involvement of reactive oxygen intermediates in spontaneous and CD95(Fas/ APO-1)-mediated apoptosis of neutrophils. Blood 89:1748-1753

6. Mountz JD, Wu J, Cheng J, Zhou T 1994 Autoimmune disease. A problem of defective apoptosis. Arthritis Rheum 37:1415-1420

7. Matute-Bello G, Liles WC, Radella F, Steinberg KP, Ruzinski JT, Jonas M, Chi EY, Hudson LD, Martin TR 1997 Neutrophil apoptosis in the acute respiratory distress syndrome. Am J Respir Crit Care Med 156:1969-1977
8. Iwai K, Miyawaki T, Takizawa T, Konno A, Ohta K, Yachie A, Seki H, Taniguchi N 1994 Differential expression of bcl-2 and susceptibility to anti-Fas-mediated cell death in peripheral blood lymphocytes, monocytes, and neutrophils. Blood 84:1201-1208

9. Liles WC, Kiener PA, Ledbetter JA, Aruffo A, Klebanoff SJ 1996 Differential expression of Fas (CD95) and Fas ligand on normal human phagocytes: implications for the regulation of apoptosis in neutrophils. J Exp Med 184:429-440

10. Chinnaiyan AM, O'Rourke K, Tewari M, Dixit VM 1995 FADD, a novel death domain-containing protein, interacts with the death domain of Fas and initiates apoptosis. Cell 81:505-512

11. Hirata H, Takahashi A, Kobayashi S, Yonehara S, Sawai H, Okazaki T, Yamamoto K, Sasada M 1998 Caspases are activated in a branched protease cascade and control distinct downstream processes in Fas-induced apoptosis. J Exp Med 187:587-600

12. Cohen GM 1997 Caspases: the executioners of apoptosis. Biochem J 326:1-16

13. Yuan J, Shaham S, Ledoux S, Ellis HM, Horvitz HR 1993 The C. elegans cell death gene ced-3 encodes a protein similar to mammalian interleukin-1 beta-converting enzyme. Cell 75:641-652

14. Enari M, Talanian RV, Wong WW, Nagata S 1996 Sequential activation of ICE-like and CPP32-like proteases during Fas-mediated apoptosis. Nature 380:723-726

15. Allgaier B, Shi M, Luo D, Koenig JM 1998 Spontaneous and Fas-mediated apoptosis are diminished in umbilical cord blood neutrophils compared with adult neutrophils. J Leukoc Biol 64:331-336

16. Koenig JM, Simon J, Anderson DC, Smith E, Smith CW 1996 Diminished soluble and total cellular L-selectin in cord blood is associated with its impaired shedding from activated neutrophils. Pediatr Res 39:616-621

17. Vasconcellos LM, Schachter AD, Zheng XX, Vasconcellos LH, Shapiro M, Harmon WE, Strom TB, Schachter D 1998 Cytotoxic lymphocyte gene expression in peripheral blood leukocytes correlates with rejecting renal allografts. Transplantation 66:562-566

18. Kumar A, Commane M, Flickinger TW, Horvath CM, Stark GR 1997 Defective TNF-alpha-induced apoptosis in STAT1-null cells due to low constitutive levels of caspases. Science 278:1630-1632

19. Muzio M, Chinnaiyan AM, Kischkel FC, O’Rourke K, Shevchenko A, Ni J, Scaffidi C, Bretz JD, Zhang M, Gentz R, Mann M, Krammer PH, Peter ME, Dixit VM 1996 FLICE, a novel FADD-homologous ICE/CED-3-like protease, is recruited to the CD95 (Fas/APO-1) death-inducing signaling complex. Cell 85:817-827

20. Watson RW, Rotstein OD, Parodo J, Bitar R, Hackam D, Marshall JC 1997 Granulocytic differentiation of HL-60 cells results in spontaneous apoptosis mediated by increased caspase expression. FEBS Lett 412:603-609

21. Sanghavi DM, Thelen M, Thornberry NA, Casciola-Rosen L, Rosen A 1998 Caspasemediated proteolysis during apoptosis: insights from apoptotic neutrophils. FEBS Lett 422:179-184

22. Jean JC, Oakes SM, Joyce-Brady M 1999 The Bax inhibitor-1 gene is differentially regulated in adult testis and developing lung by two alternative TATA-less promoters. Genomics 57:201-208

23. Aggarwal S, Gupta A, Nagata S, Gupta S 1997 Programmed cell death (apoptosis) in cord blood lymphocytes. J Clin Immunol 17:63-73

24. Hu S, Snipas SJ, Vincenz C, Salvesen G, Dixit VM 1998 Caspase-14 is a novel developmentally regulated protease. J Biol Chem 273:29648-29653

25. Woo M, Hakem R, Soengas MS, Duncan GS, Shahinian A, Kagi D, Hakem A, McCurrach M, Khoo W, Kaufman SA, Senaldi G, Howard T, Lowe SW, Mak TW 1998 Essential contribution of caspase-3/CPP32 to apoptosis and its associated nuclear changes. Genes Dev 12:806-819

26. Watson RW, Rotstein OD, Parodo J, Jimenez M, Soric I, Bitar R, Marshall JC 1997 Impaired apoptotic death signaling in inflammatory lung neutrophils is associated with decreased expression of interleukin-1 beta converting enzyme family proteases (caspases). Surgery 122:163-171

27. Yamashita K, Takahashi A, Kobayashi S, Hirata H, Mesner PWJ, Kaufmann SH, Yonehara S, Yamamoto K, Uchiyama T, Sasada M 1999 Caspases mediate tumor necrosis factor-alpha-induced neutrophil apoptosis and downregulation of reactive oxygen production. Blood 93:674-685

28. Kuida K, Zheng TS, Na S, Kuan C, Yang D, Karasuyama H, Rakic P, Flavell RA 1996 Decreased apoptosis in the brain and premature lethality in CPP32-deficient mice. Nature 384:368-372

29. Slee EA, Harte MT, Kluck RM, Wolf BB, Casiano CA, Newmeyer DD, Wang HG, Reed JC, Nicholson DW, Alnemri ES, Green DR, Martin SJ 1999 Ordering the cytochrome $c$-initiated caspase cascade: hierarchical activation of caspases-2, -3, -6, $-7,-8$, and -10 in a caspase-9-dependent manner. J Cell Biol 144:281-292

30. Whyte MKB, Meagher LC, MacDermot J, Haslett C 1993 Impairment of function in aging neutrophils is associated with apoptosis. J Immunol 150:5124-5134

31. Wen LP, Fahrni JA, Troie S, Guan JL, Orth K, Rosen GD 1997 Cleavage of focal adhesion kinase by caspases during apoptosis. J Biol Chem 272:26056-26061

32. Na S, Chuang TH, Cunningham A, Turi TG, Hanke JH, Bokoch GM, Danley DE 1996 D4-GDI, a substrate of CPP32, is proteolyzed during Fas-induced apoptosis. J Biol Chem 271:11209-11213

33. Kothakota S, Azuma T, Reinhard C, Klippel A, Tang J, Chu K, McGarry TJ, Kirschner MW, Koths K, Kwiatkowski DJ, Williams LT 1997 Caspase-3-generated fragment of gelsolin: effector of morphological change in apoptosis. Science 278:294-298

34. Howard T, Chaponnier C, Yin H, Stossel T 1990 Gelsolin-actin interaction and actin polymerization in human neutrophils. J Cell Biol 110:1983-1991

35. Merry C, Puri P, Reen DJ 1998 Phosphorylation and the actin cytoskeleton in defective newborn neutrophil chemotaxis. Pediatr Res 44:259-264

36. Martin SJ, Finucane DM, Amarante-Mendes GP, O'Brien GA, Green DR 1996 Phosphatidylserine externalization during CD95-induced apoptosis of cells and cytoplasts requires ICE/CED-3 protease activity. J Biol Chem 271:28753-28756

37. Zheng TS, Schlosser SF, Dao T, Hingorani R, Crispe IN, Boyer JL, Flavell RA 1998 Caspase-3 controls both cytoplasmic and nuclear events associated with Fas-mediated apoptosis in vivo. Proc Natl Acad Sci U S A 95:13618-13623 